\title{
Knowledge of Cervical Cancer and Acceptability of Prevention Strategies Among Human Papillomavirus-Vaccinated and Human Papillomavirus-Unvaccinated Adolescent Women in Eldoret, Kenya
}

\author{
Anisa Mburu, ${ }^{1}$ Peter Itsura, ${ }^{2}$ Hillary Mabeya, ${ }^{2}$ Alice Kaaria, ${ }^{3}$ and Darron R. Brown ${ }^{4, *}$
}

\begin{abstract}
Cervical cancer is a critical public health concern in sub-Saharan Africa. Adolescents are key targets in primary prevention strategies. Following a human papillomavirus (HPV) vaccination initiative (Gardasil) in Eldoret, Kenya, the knowledge and source of information of cervical cancer and acceptance of prevention strategies among vaccinated and unvaccinated adolescents were evaluated. A cross-sectional comparative study enrolled 60 vaccinated and 120 unvaccinated adolescent women. Institutional ethical approval was obtained and signed consent was obtained from the parents. Data collection was performed using interviewer-administered questionnaires derived from factual statements based on information from print material used for community sensitization on cervical cancer. The median age of the participants was 14.0 years (interquartile range $[\mathrm{IQR}]=13.0-15.0)$. Of 60 vaccinated adolescents, 56 (93.3\%) had heard of the HPV vaccine compared with 6 (5\%) of unvaccinated participants $(p<0.001)$. Of 60 vaccinated participants, 58 (96.7\%) had heard of cervical cancer compared with 61 $(50.8 \%)$ unvaccinated participants ( $p<0.001$ ). Both cohorts identified the school as the main source of information for cervical cancer. The two groups also showed similarity in their selection of cervical cancer prevention strategies acceptable to them such as delaying sexual debut, limiting number of sexual partners, and use of condoms for protection against sexually transmitted infections. Of 120 unvaccinated participants, 63.7\% expressed willingness to be vaccinated. Exposure to the HPV vaccine was associated with a higher knowledge of cervical cancer. The adolescents predominantly rely on the school for health information. Both cohorts of adolescents showed remarkable acceptability for cervical cancer prevention strategies.
\end{abstract}

Keywords: acceptability; adolescents; HPV vaccine; Kenya; knowledge

\section{Introduction}

Cancer of the cervix has ascended the oncological ladder to be the primary fatal gynecological cancer in developing countries. ${ }^{1}$ In 2012, 270,000 women died from cervical cancer with $85 \%$ from low- and middle- income countries. There were 530,000 new cases in 2012 worldwide. $^{2}$ Cancer of the cervix is increasingly becoming a public health concern in sub-Saharan Africa; it ranks as the first cause of female cancers in Kenya, and is the leading cause of cancer deaths/

\footnotetext{
${ }^{1}$ Department of Reproductive Health, Moi Teaching and Referral Hospital, Eldoret, Kenya.

${ }^{2}$ Department of Reproductive Health, Moi University, Eldoret, Kenya.

${ }^{3}$ Reproductive Health Services, Nairobi, Kenya.

${ }^{4}$ Department of Medicine, Indiana University School of Medicine, Indianapolis, Indiana.

*Address correspondence to: Darron R. Brown, MD, MPH, Department of Medicine, Indiana University School of Medicine, 545 Barnhill Drive, Indianapolis, IN 46202-5114, E-mail: darbrow@iu.edu

This study was presented as a poster in the AORTIC 2017 conference in Rwanda and the UNESCO-MARS 2017 Summit in Mauritius and as an oral presentation at the 2018 World Cancer Congress.
}

(c) Anisa Mburu et al. 2019 Published by Mary Ann Liebert, Inc. This Open Access article is distributed under the terms of the Creative Commons License (http://creativecommons.org/licenses/by/4.0), which permits unrestricted use, distribution, and reproduction in any medium, provided the original work is properly cited. 
mortality among all cancers in women. ${ }^{1,2}$ Annually, the estimated number of cervical cancer cases in Kenya is 5250 , whereas the mortality is $3286 .^{3}$

Human papillomavirus (HPV), a sexually transmitted virus, is the causative agent of cervical cancer with the highly oncogenic HPV types 16 and 18 present in $>90 \%$ of invasive cervical carcinoma specimen in Western Kenya. ${ }^{4,5}$ In sexually active adolescents, HPV prevalence is high with $50-80 \%$ of adolescents having active infections within 12 months of sexual debut. ${ }^{6}$

It follows that the contemporary approach is shifting toward primary prevention strategies with principal emphasis on adolescents to foster early behavioral modification including but not limited to delaying sexual debut, restricting number of sexual partners, consistent correct condom use, and not engaging in smoking. ${ }^{7}$ Recent scientific advances also evidenced the inception of HPV vaccines that have proved effective in the prevention of HPV infection and associated disease with many nations incorporating it in their national adolescent vaccination schedule.

The success of these primary cervical cancer prevention strategies is related to the level of awareness and knowledge regarding various aspects of the disease and vaccine. ${ }^{8}$ A study in Kenya showed that most Kenyan women had very little knowledge about HPV and HPV vaccination. Some women confused HPV with HIV or hepatitis B. They were willing to let their daughters be vaccinated if it meant prophylaxis against cancer; however, they stated that they would prefer a more inexpensive option with fewer dosages. ${ }^{9}$

However, there are a dearth of data on the level of awareness of cervical cancer among Kenyan adolescents and their acceptance of cervical cancer prevention strategies. Therefore, a study was conducted to compare the knowledge and source of information of cervical cancer and acceptability of prevention strategies among vaccinated and unvaccinated adolescent girls after a HPV vaccination initiative in Eldoret, Kenya that was carried out by Gardasil Access Program, under Merck and Co., Inc., in 2012 to 2013.

\section{Methods}

Cohort enrollment

This cross-sectional comparative study was carried out between May and October 2016 in six randomly selected public schools that had participated in the Gardasil Access Program's hospital-based HPV vaccination initiative in Eldoret, Kenya 3 years before this study was conducted. Using lists from each school with the names of the vaccinated adolescents, we randomly selected a total of 60 vaccinated adolescents by proportionate allocation from 3 of the schools. For the comparative cohort, we selected 120 unvaccinated adolescents from the remaining 3 schools also by proportionate allocation. The eligibility criteria included being $12-18$ years of age at the time of enrollment ( 3 years after the vaccination initiative) and being female gender; this is in accordance to the World Health Organization definition of an adolescent (10-19 years).

\section{Ethical issues}

The Moi University and Moi Teaching and Referral Hospital Institutional Research and Ethics Committee in Eldoret, Kenya approved the study protocol. As all participants were minors ( $<18$ years) in accordance to the Kenyan constitution, written informed consent was required from each parent/guardian with subsequent participant informed assent.

\section{Study procedures}

After enrolment, the adolescents had face-to-face interviews with the principal investigator and trained research assistants in classrooms or under tree shades depending on the participant's preference in a bid to create an informal set up. The interviews were carried out in English, as it is the primary language for communication at all schools in Eldoret, covering demographic details, questions on HPV transmission, and identification of cervical cancer symptoms and treatment options. The questions were based on print material used to disseminate information about cervical cancer. Each question had three choices: "Yes," "No," and "Don't know" (Supplementary Table S1). The adolescents were also asked to select cervical cancer screening modalities that they found acceptable to them with future intentions for uptake.

\section{Statistical analysis}

Categorical variables were summarized using frequencies and the corresponding percentages. Continuous variables were summarized using mean and the corresponding standard deviation if the Gaussian assumptions were holding. The median and the corresponding interquartile range (IQR) whenever the Gaussian assumptions were violated. Gaussian assumptions were assessed using Shapiro-Wilk test, the normal probability plots and histograms.

Proportions were compared using Pearson's chisquared test. However, whenever the chi-squared assumptions were violated, Fisher's exact test was used. 
Normally distributed continuous variables were compared between two levels of a categorical variable using independent sample $t$-test or one-way analysis of variance test between more than two levels of a categorical variable. The two-sample Wilcoxon rank sum test was used to compare two continuous variables whenever the Gaussian assumptions were violated. The knowledge score was compared between the vaccinated and the unvaccinated using a linear regression model adjusting for potential confounding variables. This was reported by the regression estimates and the corresponding 95\% confidence intervals (CIs). The statistical package used for data analysis was carried out using R: A Language and Environment for Statistical Computing for summarizing and comparing the variables for significant statistical inference. ${ }^{10}$

\section{Results}

Demographics

The median age of all 180 participants was 14.0 years $(\mathrm{IQR}=13.0-15.0)$ with a range of $12.0-18.0$ years. Eighteen of 180 girls $(10 \%)$ were in grade $6(12-13$ years of age), 56 of 180 girls (31.1\%) were in grade 7 (13-14 years of age), and 106 of 180 girls (58.9\%) were in grade 8 (14-15 years of age). No vaccinated girls were in grade 6 . Forty-nine of the $60(81.7 \%)$ vaccinated adolescents were in the eighth grade with the mean age for vaccination being 14 years.

\section{Knowledge}

Of the vaccinated adolescents, $96.7 \%(58 / 60)$ had heard of cervical cancer as compared with $50.8 \%(61 / 120)$ of the unvaccinated adolescents $(p<0.001)$. A majority of the vaccinated adolescents $(93.3 \%, 56 / 60)$ had heard of the HPV vaccine, whereas only $5 \%(6 / 120)$ of the unvaccinated adolescents had heard of the vaccine $(p>0.001)$. HPV was correctly identified as a sexually transmitted virus by $41.7 \%(25 / 60)$ vaccinated adolescents but only by $5 \%(6 / 120)$ unvaccinated adolescents $(p<0.001)$.

In terms of knowledge of risk factors for cervical cancer, 93.3\% (56/60) vaccinated girls knew that an early sexual debut was a predisposing factor for cervical cancer, compared with $70.8 \%$ (85/120) of unvaccinated girls $(p=0.001)$. There was no statistically significant difference in knowledge between the vaccinated and unvaccinated adolescents in selecting other risk factors for cervical cancer such as smoking and having multiple sexual partners (Table 1).

There was no significant difference between vaccinated and unvaccinated participants in awareness that
Table 1. Comparison of Knowledge of Cervical Cancer, Human Papillomavirus and its Risk Factors

\begin{tabular}{|c|c|c|c|}
\hline \multirow[b]{3}{*}{ Variable } & \multicolumn{2}{|c|}{ Vaccinated } & \multirow[b]{3}{*}{$p^{a}$} \\
\hline & $\begin{array}{c}\text { No } \\
(N=120)\end{array}$ & $\begin{array}{c}\text { Yes } \\
(N=60)\end{array}$ & \\
\hline & \multicolumn{2}{|c|}{ Participants, $n$ (\%) } & \\
\hline \multicolumn{4}{|l|}{ General knowledge } \\
\hline Heard of cervical cancer (yes) & $61(50.8)$ & $58(96.7)$ & $<0.001$ \\
\hline Heard of HPV vaccine (yes) & $6(5.0)$ & $56(93.3)$ & $<0.001$ \\
\hline \multicolumn{4}{|l|}{ HPV transmission } \\
\hline Sexual contact (correctly responded yes) & $6(5.0)$ & $25(41.7)$ & $<0.001$ \\
\hline $\begin{array}{l}\text { Blood transfusion (incorrectly } \\
\text { responded yes) }\end{array}$ & $6(5.0)$ & $8(13.3)$ & 0.074 \\
\hline Don't know & $108(90.0)$ & $27(45.0)$ & $<0.001$ \\
\hline \multicolumn{4}{|l|}{ Cervical cancer risk factors ${ }^{b}$} \\
\hline $\begin{array}{l}\text { Early sexual debut } \\
\text { (correctly responded yes) }\end{array}$ & $85(70.8)$ & $56(93.3)$ & 0.001 \\
\hline Smoking (correctly responded yes) & $62(51.7)$ & $33(55.0)$ & 0.792 \\
\hline $\begin{array}{l}\text { Multiple sexual partners (correctly } \\
\text { responded yes) }\end{array}$ & $90(75.0)$ & $49(81.7)$ & 0.414 \\
\hline $\begin{array}{l}\text { Male partner with multiple partners } \\
\text { (correctly responded yes) }\end{array}$ & $101(84.2)$ & $49(81.7)$ & 0.832 \\
\hline
\end{tabular}

${ }^{\text {a }}$ Significance is at $p \leq 0.05$.

${ }^{\mathrm{b}}$ Correctly answered yes.

cervical cancer is minimally symptomatic in its early stages (Table 2). A majority of the vaccinated adolescents correctly identified chemotherapy, radiation, or surgery as viable treatment modalities for cervical cancer $(p<0.001)$ (Table 2).

Table 2. Comparison of Knowledge of Symptom Profile, Treatment, and Screening Modalities for Cervical Cancer

\begin{tabular}{|c|c|c|c|}
\hline \multirow[b]{3}{*}{ Variable } & \multicolumn{2}{|c|}{ Vaccinated } & \multirow[b]{3}{*}{$p^{a}$} \\
\hline & $\begin{array}{l}\text { No } \\
(N=120)\end{array}$ & $\begin{array}{c}\text { Yes } \\
(N=60)\end{array}$ & \\
\hline & \multicolumn{2}{|c|}{ Participants, $n$ (\%) } & \\
\hline \multicolumn{4}{|c|}{$\begin{array}{l}\text { Correct responses for symptom profile of cervical cancer } \\
\text { Early stage (correctly responded yes) }\end{array}$} \\
\hline \multicolumn{4}{|l|}{ Late stage (correctly responded yes) } \\
\hline Lower abdominal pain & $69(57.5)$ & $47(78.3)$ & 0.010 \\
\hline Vaginal bleeding & $73(60.8)$ & $38(63.3)$ & 0.871 \\
\hline Vaginal discharge & $75(62.5)$ & $35(58.3)$ & 0.705 \\
\hline Anemia & $43(35.8)$ & $26(43.3)$ & 0.416 \\
\hline Postcoital bleeding & $61(50.8)$ & $37(61.7)$ & 0.224 \\
\hline Urine and fecal incontinence & $38(31.7)$ & $30(50.0)$ & 0.026 \\
\hline Weakness & $78(65.0)$ & $45(75.0)$ & 0.234 \\
\hline \multicolumn{4}{|l|}{ Correct responses for treatment options } \\
\hline Chemotherapy (correctly responded yes) & $22(18.3)$ & $46(76.7)$ & $<0.001$ \\
\hline Radiation (correctly responded yes) & $9(7.5)$ & $29(48.3)$ & $<0.001$ \\
\hline Surgery (correctly responded yes) & $18(15.0)$ & $36(60.0)$ & $<0.001$ \\
\hline Don't know & $79(65.8)$ & $5(8.3)$ & $<0.001$ \\
\hline \multicolumn{4}{|l|}{ Correct responses for screening options } \\
\hline Blood tests (correctly responded no) & $40(33.3)$ & $32(53.3)$ & 0.015 \\
\hline Pap smear (correctly responded yes) & $11(9.2)$ & $10(16.7)$ & 0.218 \\
\hline VIA (correctly responded yes) & $13(10.8)$ & 7 (11.7) & $>0.999$ \\
\hline Don't know & $60(50.0)$ & $21(35.0)$ & 0.080 \\
\hline
\end{tabular}

${ }^{\text {a }}$ Significance is at $p \leq 0.05$.

VIA, visual inspection with acetic acid. 
Regarding knowledge of screening options, 35\% (21/ $60)$ from the vaccinated cohort and 50\% (60/120) from the unvaccinated cohort indicated that they did not know the cervical cancer screening modalities available but there was no significant difference between the two groups. For the adolescents who correctly selected Pap smear and visual inspection with acetic acid as screening modalities, $28.4 \%(17 / 60)$ were from the vaccinated group, whereas $20 \%(24 / 120)$ were from the unvaccinated group, also with no significant difference between the two groups. Blood tests were ruled out as cervical cancer screening modalities by $53.3 \%(32 / 60)$ of the vaccinated adolescents and by $33.3 \%(40 / 120)$ of unvaccinated adolescent girls $(p=0.015)$ (Table 2).

There was no statistical difference between the vaccinated and unvaccinated adolescents in their opinion that cervical cancer was preventable.

In multivariate analysis, adolescents who were vaccinated were more likely to be knowledgeable about cervical cancer than those in the unvaccinated counterparts, regardless of the current grade of school (adjusted odds ratio $=14.4 ; 95 \% \mathrm{CI}=12.2-16.7$ ) (Table 3).

\section{Source of information}

The potential sources of information the adolescents relied on to obtain information about cervical cancer were categorized to include the school, health care workers, social media, and community outreach programs. Both cohorts selected the school as the main source of information for cervical cancer (Table 4). Despite the vaccination process being hospital based, only $22.4 \%$ $(13 / 60)$ of the vaccinated adolescents reported having heard about cervical cancer from a doctor.

\section{Acceptance of cervical cancer prevention strategies}

An assessment of the individual perceived risk of infection by HPV showed that $53.3 \%$ (32/60) of the vaccinated participants reported a higher sense of self-perceived risk compared with $22.5 \%(27 / 120)$ of the unvaccinated cohort $(p<0.001)$ (Table 5).

Table 3. Predictors of Knowledge of Cervical Cancer Derived by Logistics Regression Analysis

\begin{tabular}{lcc}
\hline Variable & $\begin{array}{c}\text { Unadjusted } \\
\text { OR }^{\mathrm{a}}(\mathbf{9 5 \%} \mathrm{CI})\end{array}$ & $\begin{array}{c}\text { Adjusted } \\
\text { OR }^{\mathrm{a}}(95 \% \mathrm{Cl})\end{array}$ \\
\hline Vaccinated & $14.7(12.6-16.8)$ & $14.4(12.2-16.7)^{\mathrm{b}}$ \\
Grade 8 & $5.3(2.5-8.1)$ & $0.7(-1.4$ to 2.9$)$
\end{tabular}

${ }^{a}$ OR: subject to logistic regression, only a positive vaccination status was the true predictor of knowledge of cervical cancer, with being a student in grade 8 not increasing the chance of being knowledgeable.

bignificance is at $p \leq 0.05$.

$\mathrm{Cl}$, confidence interval; OR, odds ratio.
Table 4. Comparison of Source of Information for Cervical Cancer Among Participants Unvaccinated and Vaccinated Against Human Papillomavirus

\begin{tabular}{|c|c|c|c|}
\hline \multirow[b]{3}{*}{ Variable } & \multicolumn{2}{|c|}{ Vaccinated } & \multirow[b]{3}{*}{$\mathbf{p}$} \\
\hline & $\begin{array}{c}\text { No } \\
(N=120)\end{array}$ & $\begin{array}{c}\text { Yes } \\
(N=60)\end{array}$ & \\
\hline & \multicolumn{2}{|c|}{ Participants, $n$ (\%) } & \\
\hline \multicolumn{4}{|l|}{ Source of information ${ }^{a}$} \\
\hline Community outreach program & $2(3.3)$ & $4(6.9)$ & 0.431 \\
\hline Doctor & $5(8.2)$ & $13(22.4)$ & 0.040 \\
\hline Social media & 19 (31.1) & $13(22.4)$ & 0.308 \\
\hline $\begin{array}{l}\text { Family member/friend } \\
\text { suffered from it }\end{array}$ & $11(18.0)$ & $11(19.0)$ & $>0.999$ \\
\hline School Health Talk & $22(36.1)$ & $20(34.5)$ & $>0.999$ \\
\hline
\end{tabular}

Significance is at $p \leq 0.05$.

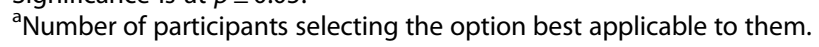

A 21 - to 30-year-old age range was selected by both the vaccinated and unvaccinated cohorts as being the most preferable for initiating a sexual debut $(p=0.791)$ rather than the 10-20 years age range or $>31$ years of age.

A high frequency of use of male condoms was also deemed acceptable by both the vaccinated $(68.3 \%$; $41 /$ $60)$ and the unvaccinated $(73.7 \% ; 87 / 120)$ adolescents.

Table 5. Comparison of Acceptability of Prevention Strategies for Cervical Cancer Among Participants Unvaccinated or Vaccinated Against Human Papillomavirus

\begin{tabular}{|c|c|c|c|}
\hline \multirow[b]{3}{*}{ Variable $^{a}$} & \multicolumn{2}{|c|}{ Vaccinated } & \multirow[b]{3}{*}{ p } \\
\hline & $\begin{array}{c}\text { No } \\
(N=120)\end{array}$ & $\begin{array}{c}\text { Yes } \\
(N=60)\end{array}$ & \\
\hline & \multicolumn{2}{|c|}{ Participants, $n$ (\%) } & \\
\hline Self-perceived risk of HPV infection & $27(22.5)$ & $32(53.3)$ & $<0.001$ \\
\hline \multicolumn{4}{|l|}{ Acceptable age of sexual debut (years) } \\
\hline $10-20$ & $2(1.7)$ & $0(0.0)$ & 0.553 \\
\hline $21-30$ & 68 (56.7) & $32(53.3)$ & 0.791 \\
\hline$\geq 31$ & $50(41.7)$ & $28(46.7)$ & 0.632 \\
\hline \multicolumn{4}{|c|}{ Acceptable frequency of using condom $(n=178)^{\mathrm{b}}$} \\
\hline Always & 87 (73.7) & $41(68.3)$ & 0.561 \\
\hline Sometimes & $21(17.8)$ & $12(20.0)$ & 0.878 \\
\hline Never & $10(8.5)$ & 7 (11.7) & 0.678 \\
\hline $\begin{array}{l}\text { Would agree to receive HPV } \\
\text { vaccine }(n=118)^{c}\end{array}$ & $75(63.6)$ & & \\
\hline $\begin{array}{l}\text { Would agree to be screened } \\
\text { for cervical cancer }\end{array}$ & $102(85.0)$ & $52(86.7)$ & 0.940 \\
\hline $\begin{array}{l}\text { Vaccination protects you from all STI so } \\
\text { no need for safe sex practices }{ }^{\mathrm{d}}(n=60)\end{array}$ & & $11(18.3)$ & \\
\hline
\end{tabular}

Significance is at $p \leq 0.05$.

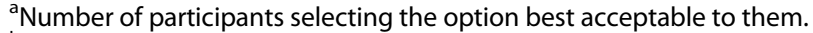

${ }^{b}$ Use of the male condom. Figures do not add up to 180 as 2 girls did not respond.

${ }^{c}$ Figures do not add up to 120 as 2 girls did not respond.

dBased on the opinion of only the girls vaccinated on the kind of protection they now have through vaccination.

STI, sexually transmitted infection. 
Seventy-five of 118 unvaccinated adolescents (63.6\%) would accept the HPV vaccine with a majority of those who would not accept it $(43.2 \%, 16 / 37)$, reporting it was because of the lack of knowledge on what it is.

Among those vaccinated, $18.3 \%$ (11/60) thought that because they were vaccinated, they were protected from all sexually transmitted infections (STIs) and so would not need to engage in safe sex practices.

A bivariate analysis tested for association between uptake of cervical cancer screening with relation to the vaccination status. As given in Table 5, similar proportions of participants among those who had been vaccinated $(86.7 \%, 52 / 60)$ and those unvaccinated against HPV $(85 \%, 102 / 120)$ would accept cervical cancer screening $(p=0.940)$.

\section{Discussion}

\section{Demographics}

Numerous studies have been carried out locally and internationally with regard to knowledge and attitudes toward HPV and HPV vaccine. ${ }^{10}$ The adolescent women in this study were matched for age, sex, and socioeconomic status by selection of schools in the same low resource setting. The defining element was vaccination status. The vaccinated adolescents were predominantly in grade 8 as they had been vaccinated 3 years before this study.

\section{Knowledge}

It was anticipated that the vaccinated adolescents would be more knowledgeable about cervical cancer and thus perceive themselves to be at a higher risk of HPV infection. ${ }^{12,13}$ In this study population, the adolescents in both groups perceived themselves to be at a higher risk of acquiring the virus but this could be attributed to the very low knowledge they had on the transmission of HPV, or to undisclosed sexual activity. Adolescents from both the vaccinated and unvaccinated cohort could identify some of the risk factors for acquisition of HPV, including an early sexual debut, multiple sexual partners, or having a sexual partner with multiple sexual partners and smoking. Knowledge of treatment and screening modalities for cervical cancer was low in both groups as seen in other adolescent populations where there is a low level of knowledge of HPV, its transmission, causes, and prevention strategies for cervical cancer among adolescents. ${ }^{14-16}$ This shows that as much as a positive vaccination status may be associated with better knowledge of cervical cancer, it does not afford sufficient awareness of the same if education offered during vaccination is not comprehensive and sufficient.

Previous studies have shown concern among parents that HPV vaccination may give the adolescent a perception of being safeguarded from all sexually transmitted illnesses and thus propelling them into promiscuity. ${ }^{17,18}$ Neither group in this study believed that the vaccine would prevent all STIs, similar to a previous study ${ }^{19}$ that strove to dispel this myth by exploring the association between getting the vaccine and engaging in risky sexual behavior, finding no such evidence. Studies looking for risky sexual behaviors after HPV vaccination found that vaccination was a good opportunity to reiterate the need for safe sex practices among adolescents and young women. ${ }^{20}$ In Western Uganda, ${ }^{21}$ it was established that HPV vaccination, knowledge and perceived sexual risk did not predict sexual behavior intentions, and with high parental involvement and communication, sexual debut could be delayed. The importance of this lies in the fact that early sexual debut and early age at first pregnancy have been linked to the development of cervical cancer later in life. ${ }^{22}$

The adolescents reported a high potential for use of condoms primarily as a protective measure against pregnancy and HIV/AIDS. Several studies locally and internationally recount several barriers for adolescents to accessing condoms such as unavailability of youthfriendly facilities providing free condoms, judgmental health practitioners, and social disapproval; common barriers experienced by adolescents in low- and middleincome countries. ${ }^{23}$ A study in Sweden showed that a school-based program promoting condom use increased the uptake of condoms for HPV prevention among adolescents. ${ }^{24}$

\section{Source of information}

There are several sources of information for HPV and cervical cancer such as social media, radio/television advertisements, print material, community outreach health talks, and information garnered from a health professional at a hospital to name but a few.

From the data accrued from the interviews, the adolescent girls in this study showed immense reliance on their teachers for information on health. This is in line with the surmise of Masika et al. ${ }^{25}$ that the empowerment of teachers would be a more feasible way to increase uptake of the vaccine through their dissemination of information on the vaccine and cervical cancer in a low resource setting such as in Eldoret, Kenya. 
Similarly, a previous study in this region ${ }^{26}$ saw the need for a collaborative effort between health workers and teachers to provide correct information while tackling stigmatism and myths that are a hindrance to the primary prevention of cervical cancer.

It is clear that adolescents need exposure to other avenues of information disbursement and need to be given access to other sources at their level. For instance, the Cancer Registry of Norway developed an application called FightHPV ${ }^{\mathrm{TM}}$, an interactive game that can be downloaded on various android and Apple devices ${ }^{27}$ by adolescents and anyone with limited knowledge of HPV and cervical cancer to learn and understand the disease and the ways to protect oneself.

\section{Acceptability}

The unvaccinated group showed great willingness to be vaccinated, which was higher compared with other studies such as that of Moroccan adolescents, which revealed that only $27 \%(282 / 1044)$ of participants were willing to accept HPV vaccination. ${ }^{28}$

The acceptability of screening for cervical cancer was remarkably high in both cohorts of adolescents despite their minimal collective knowledge of screening modalities available. This was higher than most studies where the adolescents showed very low levels of desire to be screened. ${ }^{13,15}$ This phenomenon could be explained by the possible exposure to community-based messaging on cervical cancer in this region urging women to go for screening thus making the adolescents realize their own susceptibility to cervical cancer.

\section{Limitations}

Because of the univariate results, the study was not powered to conduct a logistic regression that sufficiently shows association and predictors. Another potential limitation is that the informal interview settings utilized may have permitted a social desirability bias. However, during the pilot phase of the project, it was noted that the participants had trouble understanding the implication of some medical terms despite attempts to simplify the language. With an interviewer administering where the participants could ask for clarification, ease in responding was noted and thus this was the mode employed.

\section{Conclusion}

Receiving the HPV vaccine was associated with a higher knowledge of cervical cancer.
The adolescent women in this study predominantly rely on their school for health information. Other sources of health information like interactive social media need to be availed to adolescents. Both cohorts of adolescents showed remarkable acceptability for cervical cancer prevention strategies. There is need to increase exposure to the vaccine that may also aid in improving knowledge of cervical cancer. Prevention strategies should be made more accessible to the adolescents to improve acceptability.

\section{Implications and Contributions}

There is high morbidity and mortality from cervical cancer in sub-Saharan Africa. Thus the need to aggressively involve adolescent women in primary prevention strategies such as increasing HPV vaccination and behavioral modifications like condom use to prevent sexually transmitted diseases, limiting number of sexual partners, and regular screening.

\section{Authors' Contribution}

A.M., conception and design, development of methodology, acquisition of data, analysis and interpretation of data, writing of article. P.I., project supervision, review, and revising of data critically for important intellectual content. H.M., project supervision, review, and revising of data critically for important intellectual content. A.K., concept design, review, and revising of data critically for important intellectual content. D.R.B., review and revising of data critically for important intellectual content, final approval of the version to be published.

\section{Author Disclosure Statement}

Dr. Brown's laboratory currently receives funding from Merck and Co., Inc. In the past, Dr. Brown has received honoraria and royalties from Merck and Co., Inc. The other authors have no potential conflicts of interest to declare.

\section{Funding Information}

This work was supported by the National Institutes of Health as part of the U54 Cervical Cancer Prevention Mentoring Core Research Grant (grant no. 233).

\section{Supplementary Material \\ Supplementary Table S1}

\section{References}

1. Black E, Richmond R. Prevention of cervical cancer in Sub-Saharan Africa: the advantages and challenges of HPV vaccination. Vaccines. 2018;6:61. 
2. Bruni L, Barrionuevo-Rosas L, Albero G, et al. Human Papillomavirus and Related Diseases in Kenya. ICO/IARC Information Centre on HPV and Cancer (HPV Information Centre), 2015. Available at: https://hpvcentre .net/statistics/reports/KEN.pdf

3. Bray F, Ferlay J, Soerjomataram I, et al. Global cancer statistics 2018: GLOBOCAN estimates of incidence and mortality worldwide for 36 cancers in 185 countries. CA Cancer J Clin. 2018;68:394-424.

4. Faridi R, Zahra A, Khan K, et al. Oncogenic potential of human papillomavirus (HPV) and its relation with cervical cancer. Virol J. 2011; 8:269.

5. Ermel A, Qadadri B, Tong Y, et al. Invasive cervical cancers in the United States, Botswana and Kenya: HPV type distribution and health policy implications. Infect Agents Cancer. 2016;11. DOI:10.1186.s13027-0160102-9

6. Moscicki A. HPV infections in adolescents. Dis Markers. 2007;23:229-234.

7. Peirson L, Fitzpatrick-Lewis D, Ciliska D, et al. Screening for cervical cancer: a systematic review and meta-analysis. Syst Rev. 2013;2:35.

8. Rashid S, Labani S, Das B. Knowledge, awareness and attitude on HPV, HPV vaccine and cervical cancer among the college students in India. PLoS One. 2016;11:e0166713.

9. Becker-Dreps S, Otieno WA, Brewer NT, et al. HPV vaccine acceptability among Kenyan women. Vaccine. 2010;28:4864-4867.

10. R: The R Project for Statistical Computing [Internet]. R-project.org. 2017. Available at: https://www.R-project.org (accessed on September 26, 2017).

11. Blackman E, Thurman N, Halliday D, et al. Multicenter study of human papillomavirus and the human papillomavirus vaccine: knowledge and attitudes among people of African Descent. Infect Dis Obstet Gynecol. 2013;2013:428582.

12. Prayudi P, Permatasari A, Winata I, et al. Impact of human papilloma virus vaccination on adolescent knowledge, perception of sexual risk and need for safer sexual behaviors in Bali, Indonesia. J Obstet Gynaecol Res 2016;42:1829-1838.

13. Blumenthal J, Frey $M$, Worley $M$, et al. Adolescent understanding and acceptance of the HPV vaccination in an underserved population in New York City. J Oncol. 2012;2012:904034.

14. Panobianco M, Lima A, Oliveira I, et al. Knowledge concerning HPV among adolescent undergraduate nursing students. 2013. Texto \& Contexto-Enfermagem. 2013;22:201-207.

15. Dell $\mathrm{D}$, Chen H, Ahmad F. et al. Knowledge about human papillomavirus among adolescents. Obstet Gynecol. 2000;96:653-656

16. Fishman J, Taylor L, Kooker P, et al. Parent and adolescent knowledge of hpv and subsequent vaccination. Pediatrics. 2014; 134:e1049-e1056.

17. Elbarazi I, Raheel H, Cummings $K$, et al. A content analysis of Arabic and English Newspapers before, during, and after the human papillomavirus vaccination campaign in the United Arab Emirates. Front Public Health. 2016;4:176.

18. Linares L, Shankar V, Diaz A, et al. Association between cumulative psychosocial risk and cervical human papillomavirus infection among female adolescents in a Free Vaccination Program. J Dev Behav Pediatr. 2015;36:620-627.
19. Vázquez-Otero C, Thompson E, Daley E, et al. Dispelling the myth: exploring associations between the HPV vaccine and inconsistent condom use among college students. Prev Med. 2016;93:147-150.

20. Mullins T, Zimet G, Rosenthal S, et al. Human papillomavirus vaccinerelated risk perceptions and subsequent sexual behaviors and sexually transmitted infections among vaccinated adolescent women. Vaccine 2016;34:4040-4045.

21. Turiho A, Muhwezi W, Okello E, et al. Human papillomavirus (HPV) vaccination and adolescent girls' knowledge and sexuality in Western Uganda: a Comparative Cross-Sectional Study. 2018. DOI:10.1371. journal.pone.0137094.

22. Louie K, de Sanjose S, Diaz M, et al. Early age at first sexual intercourse and early pregnancy are risk factors for cervical cancer in developing countries. Br J Cancer. 2009;100:1191-1197.

23. Chandra-Mouli V, McCarraher D, Phillips S, et al. Contraception for adolescents in low and middle income countries: needs, barriers, and access. Reprod Health. 2014;11:1.

24. Grandahl M, Rosenblad A, Stenhammar C, et al. School-based intervention for the prevention of HPV among adolescents: a cluster randomized controlled study. BMJ Open. 2016;6:e009875.

25. Masika M, Ogembo J, Chabeda S, et al. Knowledge on HPV vaccine and cervical cancer facilitates vaccine acceptability among school teachers in Kitui County, Kenya. PLoS One. 2015;10:e0135563.

26. Vermandere $\mathrm{H}$, Naanyu $\mathrm{V}$, Degomme $\mathrm{O}$, et al. Implementation of an HPV vaccination program in Eldoret, Kenya: results from a qualitative assessment by key stakeholders. BMC Public Health. 2015;15:875.

27. Fight HPV on the App Store [Internet]. App Store. 2017. Available at: https://itunes.apple.com/sg/app/fight-hpv/id1091559571?mt=8 (accessed April 25, 2018).

28. Zouheir Y, Daouam S, Hamdi S, et al. Knowledge of human papillomavirus and acceptability to vaccinate in adolescents and young adults of the Moroccan Population. J Pediatr Adolesc Gynecol. 2016;29:292-298.

Cite this article as: Mburu A, Itsura P, Mabeya $\mathrm{H}$, Kaaria A, Brown DR (2019) Knowledge of cervical cancer and acceptability of prevention strategies among human papillomavirus-vaccinated and human papillomavirus-unvaccinated adolescent women in Eldoret, Kenya, BioResearch Open Access 8:1, 139-145, DOI: 10.1089/biores.2019.0007.

$\begin{aligned} & \text { Abbreviations Used } \\ \mathrm{Cl} & =\text { confidence interval } \\ \mathrm{HPV} & =\text { human papillomavirus } \\ \mathrm{IQR} & =\text { interquartile range } \\ \mathrm{OR} & =\text { odds ratio } \\ \mathrm{STI} & =\text { sexually transmitted infection }\end{aligned}$

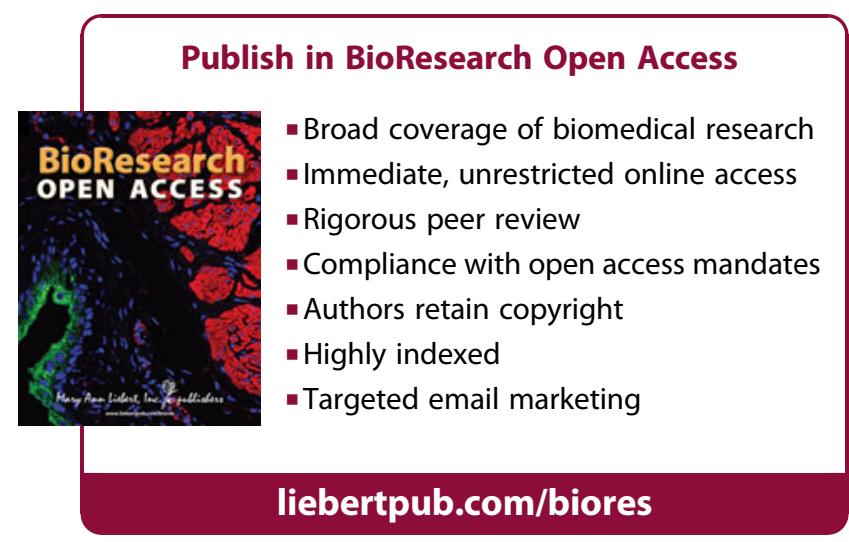

